\title{
INVESTIGATION OF THE IMPACT OF ITRF2014/IGS14 ON THE POSITIONS OF THE REFERENCE STATIONS IN EUROPE
}

\author{
Mariusz FIGURSKI * and Grzegorz NYKIEL \\ Faculty of Civil and Environmental Engineering, Gdansk University of Technology, \\ ul. G. Narutowicza 11/12, 80-233 Gdansk, Poland \\ *Corresponding author's e-mail: mariusz.figurski@pg.edu.pl
}

\begin{tabular}{l} 
ARTICLE INFO \\
\hline Article history: \\
Received 31 May 2017 \\
Accepted 25 August 2017 \\
Available online 7 September 2017 \\
\hline
\end{tabular}

Keywords:

International Terrestrial Reference Frame Global Navigation Satellite System Antenna calibration

IGS14

IGb08

\begin{abstract}
In January 2017, the new realization of the International Terrestrial Reference Frame (ITRF) ITRF2014, was released. Besides the higher accuracy and consistency of the new frame, an entirely new element - the post seismic deformation - was included. Moreover, the definition of the fundamental points has also been changed. All changes related to the new reference frame haveconsequences in the precise positioning using GNSS observations. In this paper, on the basis of results and analysis obtained from Bernese 5.2 software package, the most important changes, related to GNSS data processing are presented. Our analysis confirmed that the differences between ITRF2008 and ITRF2014 are minor. However, changing GNSS antenna calibrations from IGb08 to IGS14 causes changes of stations coordinates up to several millimeters, especially for the vertical component. This effect is mainly due to the introduction of new or updated absolute antenna calibrations. Such changes of coordinates have also impact on the realization of the European Terrestrial Reference Frame (ETRF).
\end{abstract}

\section{INTRODUCTION}

The determination of objects' location is one of the primary tasks in geodesy. The position of any object is always determined in the defined reference frame. There is no absolute reference frame and therefore point localization depends on the adopted convention. Even in case of techniques defined as absolute, the determined value has to be referred, e.g. the position determination with Precise Point Positioning (PPP) approach is expressed in a frame realized by satellite orbits. Geometric location of points on the Earth's surface, as well as satellite positions, are determined in the geocentric frame(conventionally it is related to the center of Earth mass), using cartesian, spherical or ellipsoidal coordinates.

The ITRFyy is a practical realization of the International Terrestrial Reference System (ITRS), based on four space geodetic techniques: Very Long Baseline Interferometry (VLBI), Satellite and Lunar Laser Ranging (SLR and LLR), Global Navigation Satellite Systems (GNSS) and Doppler Orbitography and Radio positioning Integrated by Satellite (DORIS). A reference frame solution - beyond the definition and estimation of the reference frame parameters (origin, orientation, scale) is practically a set of point coordinates and velocities together with the full variance-covariance matrix. The frame orientation is determined with respect to the International Celestial Reference Frame (ICRF), based on signal analysis from distant celestial objects (radio sources in VLBI technique). None of these techniques can independently provide correct definition of all parameters, which define the reference system. The ITRF as a compilation of advantages of all space geodetic techniques is characterized by the highest currently available accuracy (Altamimi et al., 2016). The ICRF and ITRF linking parameters, known as EOP (Earth Orientation Parameters), allow for determination of momentary position of Earth's rotation axis. These are: UT1 universal time or LOD (Length Of Day), which describe the time of a single rotation of the Earth in relation to its momentary rotation axis, the CEP (Celestial Ephemerides Pole) coordinates and the factors that allow for including the precession and nutation parameters (Seidelmann, 1982).

\subsection{ITRF2014 CHARACTERIZATION}

Twelve ITRS realizations have been published until 2017 (Altamimi et al., 2007; Altamimi et al., 2011; Altamimi et al., 2016). Each subsequent realizations were based on all available observations and the latest version of the frame is always 
considered as a refinement of previous ITRS realizations (Altamimi et al., 2016). ITRF2014, as the most recent solution is based on the homogeneous reprocessing of each of the four techniques observations (Altamimi et al., 2016), where the maximum time series available for each geodetic technique were used. In case of the International VLBI Service (IVS) these were 5789 session-wise solutions, the SLR contribution by the International Laser Ranging Service (ILRS) involved 1391 solutions, the International GNSS Service (IGS)provided 7714 solutions (second reprocessing of GPS and GLONASS observations), whereas in case of International DORIS Service (IDS) 1140 DORIS solutions (Altamimi et al., 2016) were used. In the definition of the ITRF2014 new local ties measurements between VLBI, SLR, GNSS and DORIS were used. The quality of these links between the different techniques is crucial for the frame accuracy, due to the fact that the combination of solutions in ITRF depends on the availability of colocation stations. Such stations are equipped with instrumentation of at least two different space geodetic techniques. The local ties between them are usually measured by classical techniques or using GNSS. In total 139 SINEX files containing measured local ties at colocation stations were used in ITRF2014. The ITRF2014 realization involved two steps. First one consisted of stacking the individual technique-dependent time series to estimate long-term cumulative solutions, which contain station position, velocity and EOP (Altamimi et al., 2007; Altamimi et al., 2011). In the second step, the results obtained in the first step were combined with the local ties at the colocation sites.

Two new innovations have been applied to the definition of the new ITRS realization. First one was modelling the periodic seasonal signals for stations with sufficient time span. Second one was the modelling of so-called post seismic deformation (PSD) for sites affected by major earthquake. The seasonal signals were modelled by adding to the coefficients of sinusoidal annual and semi-annual functions to the combination model. The PSD were modelled (before the stacking), by applying parametric models that were fitted to ITRF2014 station position time series (Altamimi et al., 2016).
Finally, the ITRF2014 is defined by the following parameters:

1. Origin: zero translation parameters at epoch 2010.0 and zero translation rates with respect to SLR solution.

2. Scale: zero scale factor at epoch 2010.0 and zero scale rate with respect to the VLBI and SLR solutions.

3. Orientation: zero rotation parameters at epoch 2010 and zero rotation rates between ITRF2008 and ITRF2014. These two conditions were applied over a set of 127 reference stations located at 125 sites.

Similarly to previous ITRS realizations, the origin of the ITRF2014 was defined solely on the basis of SLR data, corresponding to zero translation and no translation changes between ITRF2014 and SLR cumulative solutions. The accuracy of the ITRF2014 origin can be assessed by referring them to the implementation of the ITRF2008. Comparison of these frames shows the presence of a small component translation for the 2010.0 epoch, which are 1.6, 1.9 and $2.4 \mathrm{~mm}$ respectively for the $\mathrm{X}, \mathrm{Y}$ and $\mathrm{Z}$ components. Changes in time of this translation are statistically zero for all three components (Altamimi et al., 2016). To determine the scale of the ITRF2014 a similar approach as for the ITRF2008, was adopted. The zero scale and no change in scale between ITRF2014 and the scale determined as the arithmetic mean of SLR and VLBI solution (Altamimi et al., 2011; Altamimi et al., 2016) were assumed. For geodetic applications, for the purpose of preserving the continuity of solutions, the relationship between ITRF2014 and previous ITRFs is crucial. Therefore, similarly as in previous ITRS realizations, 14parametric transformations with respect to ITRF2008 and previous ones, have been derived (Table 1). To do this, the same set of 127 stations which were used to align orientation parameters and their temporal variation between ITRF2014 and ITRF2008 were used. The main criterion of these 127 stations choice was to provide the best possible spatial distribution, using as many VLBI, SLR, DORIS and GNSS solutions as possible and ensuring the best compatibility between frames.

Table 1 Transformation parameters and their rates between ITRF2014 and ITRF2008 at epoch 2010.0 (Altamimi et al., 2016). Transformation coefficients, their WRMS, coefficients rates, and their WRMS are shown in the following rows.

\begin{tabular}{|c|c|c|c|c|c|c|c|}
\hline & TX & TY & $\mathbf{T Z}$ & DS & $\mathbf{R X}$ & RY & RZ \\
\hline & {$[\mathrm{mm}]$} & {$[\mathrm{mm}]$} & {$[\mathrm{mm}]$} & $\mathrm{ppb}$ & mas & mas & mas \\
\hline & 1.6 & 1.9 & 2.4 & -0.02 & 0.0 & 0.0 & 0.0 \\
\hline$+/-$ & 0.2 & 0.1 & 0.1 & 0.02 & 0.006 & 0.006 & 0.006 \\
\hline RATES & 0.0 & 0.0 & -0.1 & 0.03 & 0.0 & 0.0 & 0.0 \\
\hline$+/-$ & 0.2 & 0.1 & 0.1 & 0.02 & 0.006 & 0.006 & 0.006 \\
\hline
\end{tabular}




\subsection{IGS FRAME}

The GNSS technique plays a very important role in practical realization of the ITRS. This is due to the large number of stations spread all over the Earth, which translates into a predominant contribution of this geodetic technique through local ties to markers of the other space geodetic techniques. However, there is only a dozen of such local ties between the other techniques (Altamimi, 2012). As a result of such poor configuration of local ties between SLR and VLBI, the IGS network plays an extremely important role in linking them together, during determination of the origin and scale of the frame (Altamimi and Collilieux, 2009). GNSS station can be found on almost all lithospheric blocks and to a large extent they belong to the IGS core network, which is used for e.g. orbit determination. The GNSS technique allows also for accurate determination of the ITRF orientation in space (in relation to the celestial frame) and for monitoring its changes, as well as for the most precise and accurate determination of the pole motion. GNSS stations allow for direct reference to ITRFyy (in real or near real time).

As already mentioned, the ITRF is based on four different techniques and it could be an optimal solution for GNSS products. However, according to the current practice the publication of a new ITRS realization is quickly followed by the release of an updated IGS reference frame solution, which benefits from the actual ITRF solution and the update of the GNSS PCV models and may be characterized by higher internal consistency and allows IGS for providing its products (coordinate and ephemeris) with the best accuracy. In 2000 the IGS97 frame, which was linked to the ITRF97 and consisted of 51 GPS permanent stations, was the first in this series. Since then, this frame was periodically updated: since 2006 the IGS05 was in force, in 2011 was changed to the IGS08 (Rebischung, 2011) and then, due to the growing number of unusable stations within IGS08 frame, the IGb08 was introduced. This frame was linked to the ITRF2008 and included new GNSS antenna calibrations. IGb08 was used in the second IGS reprocessing campaign. In 2017, in parallel with the redefinition of the ITRF, a new and revised IGS14 frame has been introduced including new and update calibration of satellite and GNSS receivers' antennas.

At the time of writing, for the most recent realization of ITRF and IGS, i.e. ITRF2014 and IGS14, the transformation parameters between them are not published because it is assumed that their global values are zero. The differences in station coordinates which are expressed in these two frames are treated as characteristic values of the stations due to the fact that the ITRF2014 includes antenna calibration values consistent with IGS08 standards, whereas IGS14 is consistent with IGS14 standards. The similar situation occurred in case of previous ITRFs (Rebischung, 2011). Adopting different standards for antenna models can lead to regional biases between frames, although the global transformations values are assumed to be zero.

\subsection{INFLUENCE OF THE ITRF2014/IGS14 REDEFINITION ON THE GNSS OBSERVATIONS PROCESSING IN EUROPE}

From the practical point of view of GNSS observations processing, the most important issue in redefining the reference frame is to ensure the continuity of coordinates between subsequent frame implementations. To do this, we must first determine the cause of possible discontinuity. Apart from the mentioned differences in the antenna calibration standards, a definition of the center of the ITRF frame may be problematic. According to the ITRF2014 arrangement described above, the center of the global frame (translation components and their derivatives over time) is derived from SLR solutions. In theory, it could also be determined by GNSS technique, but the accuracy in this case would be lower. In the IGS14 frame, which is being used for the weekly combined solutions of the European GNSS Network (EPN), the parameters of the ITRF2014 frame were adopted. However, the actual center of the IGS14 frame estimated from GNSS processing is not consistent with ITRF2014. Moreover, the translation component is closely related to GNSS orbit modeling (Hugentobler, 2005). All orbit modelling errors are reflected in the origin of the frame, which (among other things) causes that GNSS measurements are not used for translation estimation. The IGS14 antenna calibrations are included in the IGS14 frame, while transformation coefficients from global to local coordinates (e.g. for the European continent) are estimated based on GNSS solutions obtained using IGS08 (or IGb08) calibrations. The selection of the way of linked local network to ITRF as well as the selection of fixed stations is equally important. Discontinuities and errors that occur on individual stations can cause an aggregate effect that changes the origin of the frame, i.e. the translation parameter (Drewes, 2007).

\section{PRACTICAL REALIZATION OF THE ITRF2014/IGS14 IN EUROPE}

During processing of GNSS observations for the last two decades, it was noticed that each ITRS realization introduce discontinuities in GPS and GPS/GLONASS solutions (Kaminski et al., 2010). This is the case for the actual ITRF2014 solution as well. As it was already mentioned, it depends on satellite ephemeris, satellite and ground antenna phase center models and station coordinates and velocities. Satellite ephemerides are always determined based on ground-based GNSS observations from selected IGS (core) stations, i.e. they are expressed in a given ITRF realization, as well as coordinates and velocities of the stations. In contrast, antenna phase center models change regardless of the redefinition of the ITRF and are, besides antenna replacement, one of the main sources of inconsistencies in maintaining the continuity of GNSS solutions over a long period of time. 


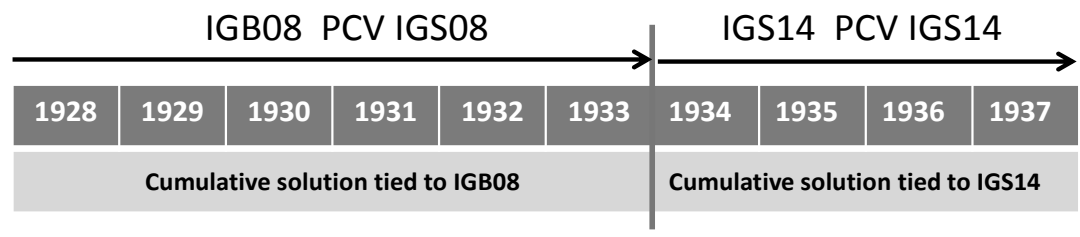

Fig. 1 Method of cumulative GNSS solutions independently from GPS weeks 1928 to 1933 (ITRF2008) and from GPS week 1934 to 1937 (ITRF2014).

In our research we made attempts to see how these factors affect the continuity of solutions in the European continent during the transition from the ITRF2008 to the ITRF2014.

For our analyses we used daily GNSS observations from $136 \mathrm{EPN}$ reference stations located throughout the European continent, i.e. approximately $50 \%$ of the currently operating GNSS stations of the EPN were included. The analyzed time span covered six weeks (GPS week1928 to 1933) where the IGb08 was still in place and four weeks after the IGS14 was introduced (GPS week1934 to 1937) (Fig. 1). All observations were processed using the Bernese GNSS software version 5.2 (Dach et al., 2015) and in accordance with the standards used by EPN Analysis Centers (EUREF 2016). To process data from GPS weeks 1928 to 1933, precise ephemerides and pole motion parameters expressed in IGb08 were used. Data from GPS weeks 1934 to 1937 were processed using precise ephemerides and pole motion parameters expressed in IGS14. In both cases the solutions from the Center for Orbit Determination in Europe (CODE) were used. Coordinates were estimated using differential approach for each day independently, without introducing conditions defining external reference frame (free network). Then, coordinates were combined (cumulated) to weekly solutions. The only external information was the orbits, which parameters were assumed to be error free and therefore were not estimated during the processing. In such case, the network geometry is determined solely on the basis of GNSS observations and is not biased by fixed station errors, which could bias the aligned network. It has to be kept in mind that solutions obtained in such way do not refer to the adopted reference frame (ITRFyy or IGSyy), but allow for detection of signals and frequencies in coordinate time series, which are identified as geophysical phenomena. A network developed in this way will exhibit significant daily shifts, i.e. the coordinates of each station estimated independently for each day will vary from each other. In our study, we used the above approach only to create normal equations, which were further combined in the weekly solution with the ADDNEQ2 program (Dach et al., 2015).

In the study of the changes which take place during redefinition of the ITRF, the way in which the processed network is fixed or constrained, is crucial. The optimal method to define a reference frame for a network is to add constraints on the Helmert transformation parameters for the coordinates of the selected network stations (core stations). These stations will define the reference frame.

In other words, the introduction of the minimum number of conditions eliminates the matrix singularity of the normal equations system. This method does not impose conditions on individual stations but on the barycenter of all fixed stations or on the orientation of the entire network. In our case we applied a condition that the barycenter of coordinates estimated from GNSS observations is identical to the barycenter of the a priori coordinates. From practical point of view, this is the condition to constrain the network translation parameters (Dach et al., 2015). The use of all seven parameters for local networks leads to too many degrees of freedom. Additionally, some parameters are correlated, this approach is not recommended. The advantage of frame realization by imposing conditions on the translation parameters of the network geometric center is that minor reference station coordinates errors do not distort the network geometry and they do not reduce the accuracy of the reference frame itself.

The influence of stations selection for linking the network to the ITRF was discussed in many studies, which concluded that the best results are obtained when large number of stations, were used (Szafranek et al., 2013; Wöppelmann et al., 2008). Taking the usage of the minimum constraint method (minimum number of conditions) into account, the reliability of GNSS network determination increases with the number of high quality fixed reference stations and their geographical range. Consequently, in our analysis, we selected 26 fixed reference stations (core stations) evenly distributed over the whole area of interest. They were characterized by the greatest coordinate stability over the considered period. The coordinates and velocities of the selected stations were determined in IGb08 and IGS14 reference frames respectively.

For the analyzed time span from GPS weeks1928 till 1937 we tested four solutions (named A-D), which differ in reference frame and antenna phase center calibrations (Table 2). Daily solutions were combined in two cumulative solutions according to the two time spans (solution 1 from GPS weeks 1928 to 1933 , solution 2 from GPS weeks 1934 to 1937). Satellites ephemeris and EOP were expressed in $\mathrm{IGb} 08$ and IGS14 respectively. 
Table 2 Types of solutions used in analysis.

\begin{tabular}{c|cc}
\hline \multirow{2}{*}{ SOLUTION } & $\begin{array}{c}\text { COORDINATES AND } \\
\text { VELOCITIES } \\
\text { OF FIXED STATIONS }\end{array}$ & $\begin{array}{c}\text { ANTENNA PHASE CENTER } \\
\text { CALIBRATIONS (PCC) }\end{array}$ \\
\hline 1A & Ephemeris $(E P H)$ and EOP - IGb08 frame (1928-1933) \\
1B & IGb08 & IGS08 \\
1C & IGS14 & IGS14 \\
1D & IGS14 & IGS08 \\
\hline & IGb08 & IGS14 \\
\hline 2A & Ephemeris (EPH) and EOP - IGS14 frame (1934-1937) \\
2B & IGb08 & IGS08 \\
2C & IGS14 & IGS08 \\
2D & IGS14 & IGS14 \\
\hline
\end{tabular}

The solutions 1 and 2 were expressed in the same reference epoch (2017.077) based on a linear model, where coordinates and velocities of the stations were the parameters. We finally obtained eight cumulative solutions, four for IGb08 reference frame (GPS week 1928-1933) and four for IGS14 (1934-1937) (for Table 2 for the details).

For all cumulative solutions the same set of 26 core stations and minimum constraints were applied to define the geodetic datum. For each solution we independently checked the quality of the frame realization by fixed stations. For this purpose we used Helmert transformation, where the primary frame was formed by a priori coordinates, and secondary frames were formed by subsequent calculations for each solution. We adopted the following acceptance criterion for the fixed station: $10 \mathrm{~mm}$ for the topocentric components $\mathrm{N}$ and $\mathrm{E}$, and $20 \mathrm{~mm}$ for $\mathrm{U}$ direction. If any station exceeded these limits it was eliminated from fixed reference set. However, in that case the actual ITRF frame implementation should have been different. Hence we selected a set of 26 fixed stations, which number is constant for all solutions and fulfilled acceptance criterion. Thus, in each solution the network is homogeneous in terms of its reference, and together with ephemeris, implements given reference frame.

When the GNSS networks are processed, it is commonly assumed that the frame is realized by satellite ephemeris, EOP, and coordinates and velocities of fixed stations. Therefore, changing the frame also involves a change of these parameters. In addition, the frame change is used for introducing new and enhanced antennas calibrations, which took place when the IGb08 or IGS14 were introduced. It is worth to notice that ITRF2014/IGS14 frame was built on the basis of solutions where IGb08 antenna calibrations were used. These calibrations differ from the IGS14 standard. This is the reason why we checked the continuity of stations before and after the introduction of a new ITRF realization. According to its definition, by transforming the coordinates from solutions $2 \mathrm{~B}$ and $1 \mathrm{~A}$ using the 7-parameter Helmert transformation, we should obtain the parameters similar to those in Table 1. In transformation between ITRF2014 and
ITRF2008 no rotation component was assumed by the authors (Altamimi et al., 2016). However, in our case we estimated this component to verify whether there is no rotation effect for the local network being a subset of the global one.

In Table 3 coefficients of transformation 2B-1A (corresponding to Table 1) are presented. It can be seen that translation for component $\mathrm{X}$ differs about 5.5 millimeters from its analogue in Table 1. For Y component these values differ approximately $4.2 \mathrm{~mm}$, while for component $\mathrm{Z}$ the difference is over $6.4 \mathrm{~mm}$. Also, the scale parameter is greater than $0.93 \mathrm{ppb}$ from the value in Table 1 . The rotation coefficients have small values that may indicate a slight rotation of the reference frame realized by the analyzed network with respect to the global ITRF. Such big differences between obtained results and these one presented by Altamimi (Table 1), are caused by three factors, two of them having a major impact. First one is the limited extension of the computed network. We used 127 stations which, even though are located throughout Europe, are only part of global network used in Altamimi transformation. Thus, a frame which is realized by regional network can be (or even should be) slightly different. The second major factor, in our opinion - the main one, is the used antenna calibrations. Altamimi in his work still used IGS08 calibrations, but together with introduction of ITRF2014 an improved solution was introduced IGS14. Thus, in our calculations we used IGS14 and IGS08 calibrations for solution 2B (IGS14 frame) and 1A (IGb08 frame) respectively. In our opinion, such approach has the biggest impact on the obtained differences in transformation coefficients. In case when the same antenna calibrations were used for both IGS14 and IGb08 solutions (2A-1A, 2B-1B), the differences between our results and Altamimi are significantly smaller: $(0.50,2.58,-1.88) \mathrm{mm}$ and $(0.11,-2.05,-2.17) \mathrm{mm}$ for translation coefficients of 2A-1A (III) and 2B-1B (IV) transformations respectively. The impact of IGS14 calibrations is closely presented in next parts of paper.

The third factor which has impact on the obtained differences is used ephemerides. In our research we did not analyze the magnitude of this 
Table 3 Helmert geocentric transformation coefficients between analyzed solutions in geocentric system (see Table 2).

\begin{tabular}{|c|c|c|c|c|c|c|c|}
\hline & TX & TY & $\mathbf{T Z}$ & DS & $\mathbf{R X}$ & RY & $\mathbf{R Z}$ \\
\hline & {$[\mathrm{mm}]$} & [mm] & [mm] & $\mathrm{ppb}$ & mas & mas & mas \\
\hline \multicolumn{8}{|c|}{ I. 2B-1A: IGS14 (EPH: IGS14; PCC: IGS14)-IGb08 (EPH: IGb08; PCC: IGS08) } \\
\hline & 7.15 & 6.16 & 8.85 & -0.95 & -0.156 & -0.062 & 0.080 \\
\hline$+/-$ & 1.07 & 1.30 & 1.02 & 0.14 & 0.039 & 0.039 & 0.035 \\
\hline \multicolumn{8}{|c|}{ II. 2A-1B: IGb08 (EPH: IGS14; PCC: IGS08)- IGS14 (EPH: IGb08; PCC: IGS14) } \\
\hline & -5.00 & -6.71 & -7.83 & 0.49 & 0.055 & -0.039 & -0.061 \\
\hline$+/-$ & 1.01 & 1.23 & 0.96 & 0.13 & 0.037 & 0.036 & 0.33 \\
\hline \multicolumn{8}{|c|}{ III. 2A-1A: IGb08 (EPH: IGS14; PCC: IGS08)-IGb08 (EPH: IGb08; PCC: IGS08) } \\
\hline & 1.10 & -0.68 & 0.52 & -0.20 & 0.037 & -0.050 & 0.004 \\
\hline III* & 1.14 & -0.68 & 0.60 & -0.20 & -0.037 & -0.050 & 0.003 \\
\hline$+/-$ & 0.84 & 1.02 & 0.80 & 0.11 & 0.030 & 0.030 & 0.027 \\
\hline \multicolumn{8}{|c|}{ IV. 2B-1B: IGS14 (EPH: IGS14; PCC: IGS14)-IGS14 (EPH: IGb08; PCC: IGS14) } \\
\hline & 1.49 & -0.15 & 0.23 & -0.24 & $\mathbf{- 0 . 0 5 7}$ & -0.034 & 0.008 \\
\hline $\mathrm{IV}^{*}$ & 1.32 & -0.06 & 0.27 & -0.24 & -0.058 & -0.034 & 0.009 \\
\hline$+/-$ & 0.083 & 1.01 & 0.79 & 0.11 & 0.030 & 0.030 & 0.027 \\
\hline \multicolumn{8}{|c|}{ V. 2D-1D: IGb08 (EPH: IGS14; PCC: IGS14)-IGb08 (EPH: IGb08; PCC: IGS14) } \\
\hline & 1.56 & -0.25 & 0.14 & -0.23 & -0.055 & -0.030 & 0.005 \\
\hline$+/-$ & 0.84 & 1.01 & 0.79 & 0.11 & 0.030 & 0.030 & 0.027 \\
\hline \multicolumn{8}{|c|}{ VI. 2C-1C: IGS14 (EPH: IGS14; PCC: IGS08)-IGS14 (EPH: IGb08; PCC: IGS08) } \\
\hline & 1.05 & -0.62 & 0.60 & -0.20 & $-\mathbf{0 . 0 3 9}$ & -0.053 & 0.005 \\
\hline$+/-$ & 0.84 & 1.02 & 0.80 & 0.11 & 0.030 & 0.030 & 0.027 \\
\hline \multicolumn{8}{|c|}{ VII. 2D-1C: IGb08 (EPH: IGS14; PCC: IGS14)-IGS14 (EPH: IGb08; PCC: IGS08) } \\
\hline & -2.02 & 0.23 & -3.63 & -0.47 & -0.110 & -0.059 & 0.045 \\
\hline$+/-$ & 1.09 & 1.23 & 1.03 & 0.14 & 0.039 & 0.039 & 0.035 \\
\hline
\end{tabular}

Helmert transformation residuals

IGS14 (EPH: IGS14; PCV: IGS14; weeks 1934-1937) - IGb08 (EPH: IGb08; PCV: IGS08; weeks 1928-1933)
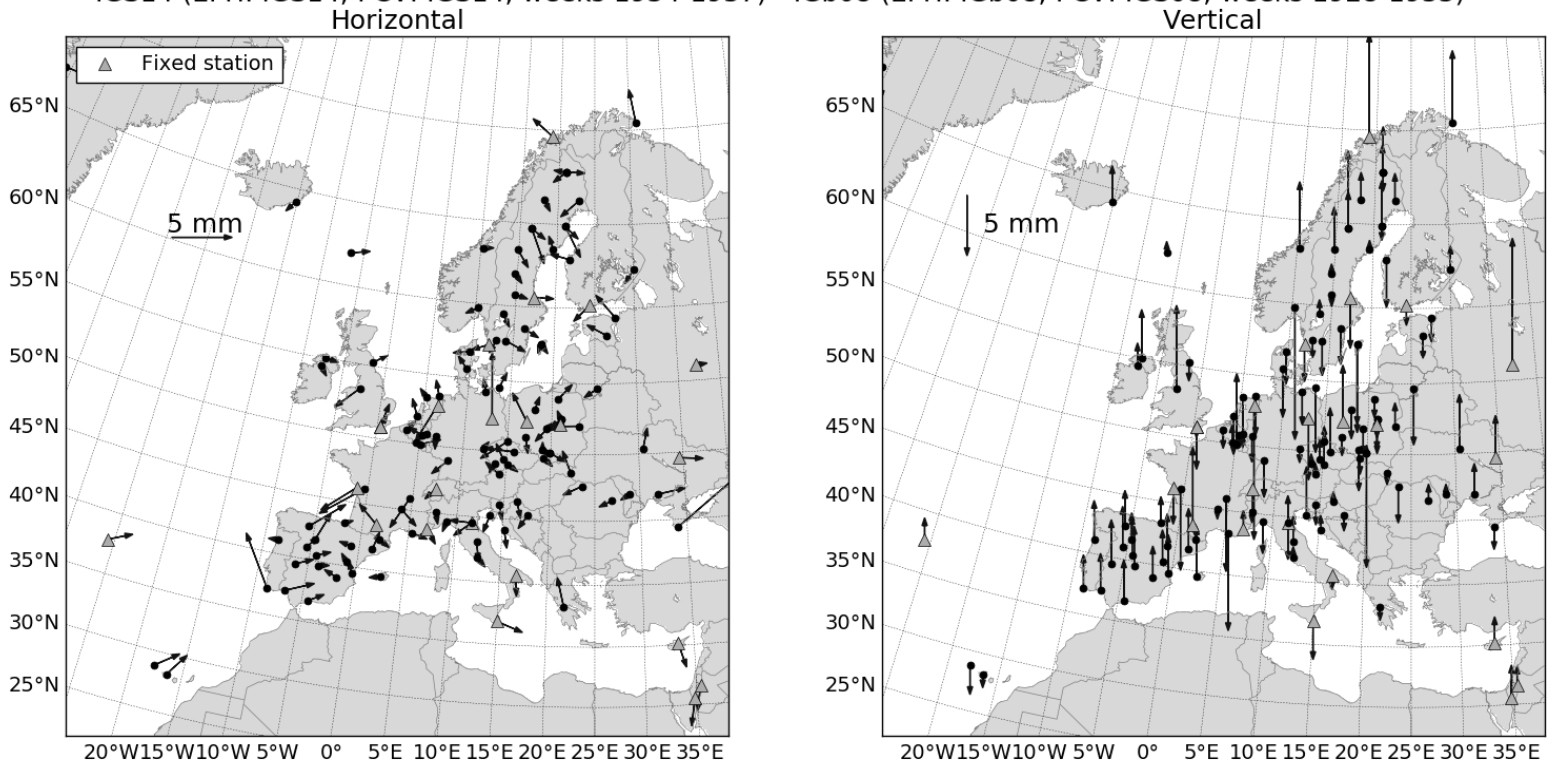

Fig. 2 Residuals of geocentric Helmert transformation between IGb08 (IGS08 antenna calibrations) and IGS14 (IGS14 antenna calibrations) frames (2B-1A transformation).

impact. To investigate it properly, new determination of old ephemerides in IGS14 frame, or ephemerides transformation from IGb08 to IGS14 should be performed.

Figure 2 shows coordinate residuals after the transformation. It is clearly seen that coordinates differences occur mainly in the height component for some stations amount up to $8 \mathrm{~mm}$. This effect is better seen in Figure 3, where differences in coordinates between solutions $1 \mathrm{~A}$ and $2 \mathrm{~B}$ are presented. The figure shows a clear systematic height bias for all stations of the analyzed network, ranging from a few 
Coordinates differences

IGS14 (EPH: IGS14; PCV: IGS14; weeks 1934-1937) - IGb08 (EPH: IGb08; PCV: IGS08; weeks 1928-1933)
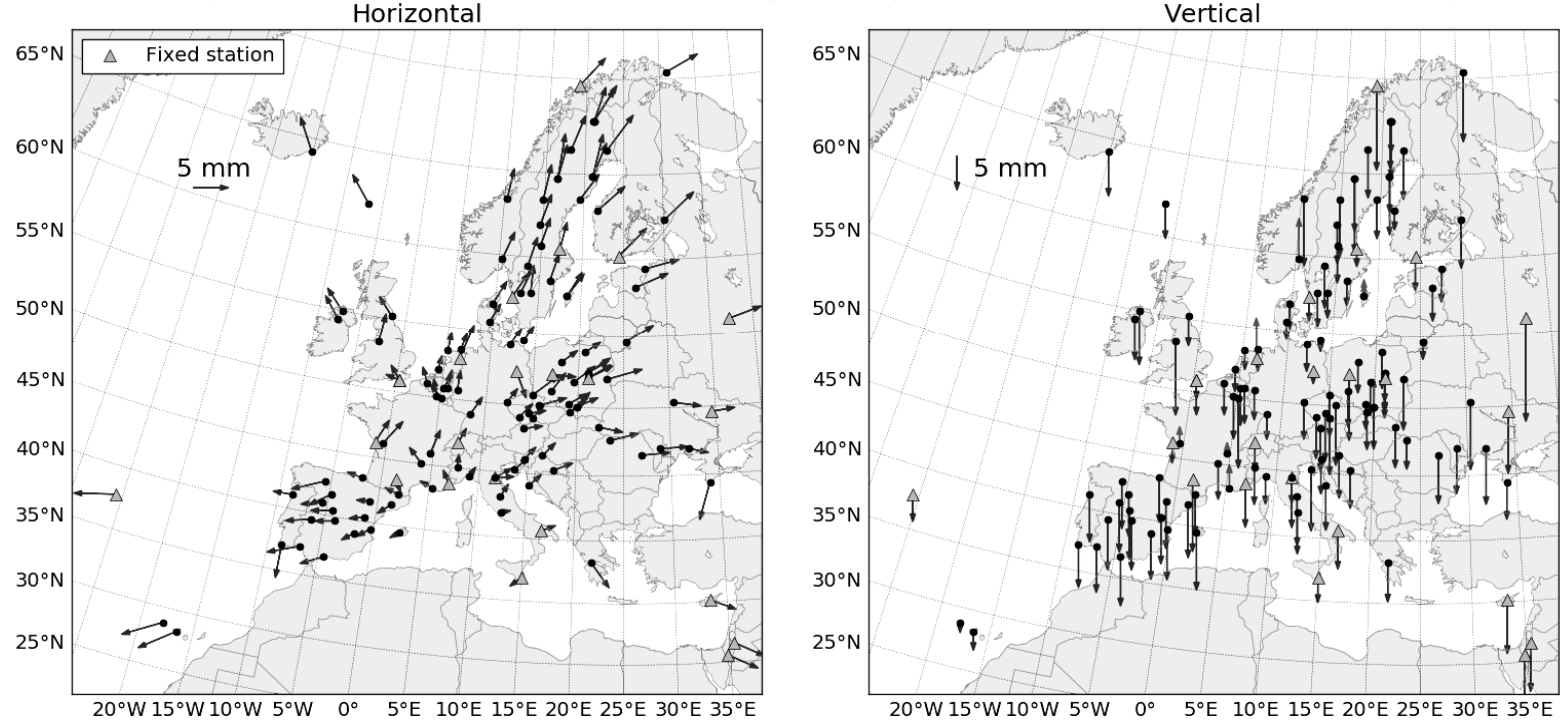

Fig. 3 Coordinates differences between IGb08 (IGS08 antenna calibrations) and IGS14 (IGS14 antenna calibrations) frames (solutions $2 \mathrm{~B}-1 \mathrm{~A})$.

$\mathrm{mm}$ up to $10 \mathrm{~mm}$. From a practical point of view, this means that stations estimated in IGS14 frame have a lower height than estimated in IGb08. This causes discontinuities in coordinates time series, especially in the height component. Figure 3 also shows the shift of horizontal coordinates, which indicates translation changing between ITRF components (Table 3). These results are only indicative. Reliable shifts can be obtained only when the calculation for longer period of time (at least a few years) will be performed.

In order to present impact of new reference frame and improved antenna models on stability of EPN stations, we show coordinate time series for two stations POTS00DEU (Potsdam, Germany) and DARE00GBR (Daresbury, UK), which antennas have been changed between IGS08.atx and IGS14.atx. At the first station individual calibration was introduced (instead of type mean) and at the second one, the improved type mean calibration was presented. For these stations we estimated coordinates in IGb08 frame with IGS08 antennas calibrations (dotted line) from 1928 to 1933 GPS week and in IGS14 frame with IGS14 antennas calibrations (solid line) from 1928 to 1937 GPS week. Geocentric coordinates were converted to the local topocentric frame (NEU).

In practice, satellite signal reception is referred to the antenna phase center point, which position is not strictly defined, but depends on the direction (azimuth and elevation angle) from which the satellite signal is received, and on the frequency of the signal (distinguishes other phase centers for different signals frequencies). The location of the phase center
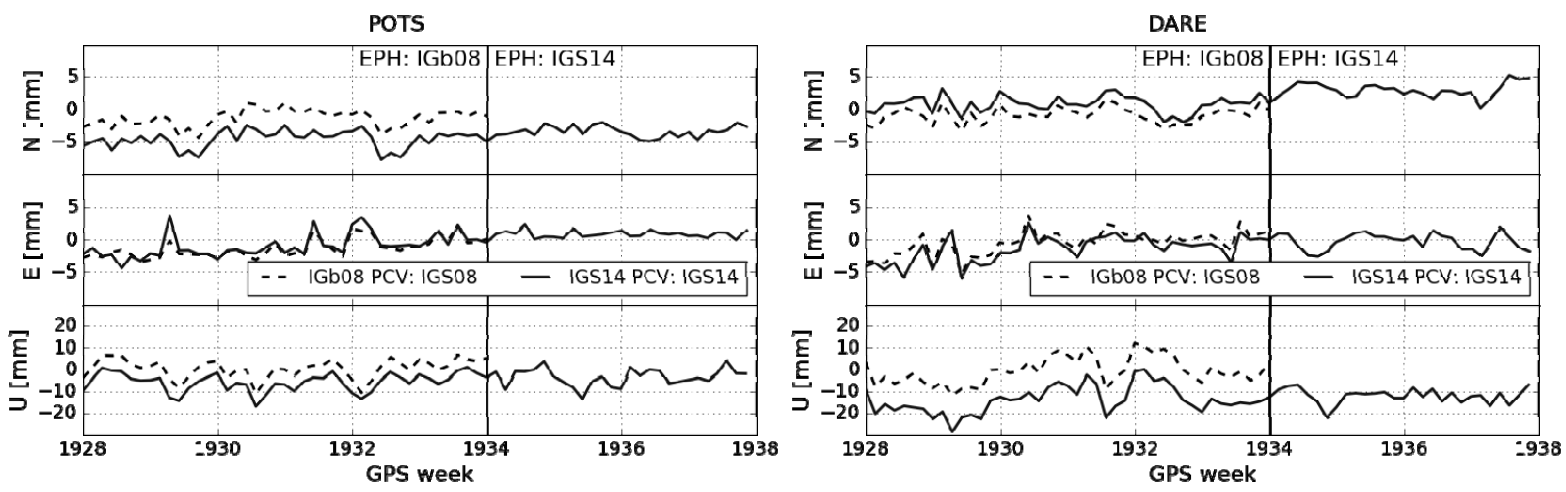

Fig. 4 Positioning results (w.r.t. IGb08 EPN cumulative solution at epoch 1934 GPS week) for stations POTS00DEU (Potsdam, Germany, left) and DARE00GBR (Daresbury, UK, right). 
LEIAR25 LEIT (igs14_1935.atx)

LEIAR25 LEIT (igs08̄_1930.atx) frequency: $\mathrm{G} \overline{0} 1$

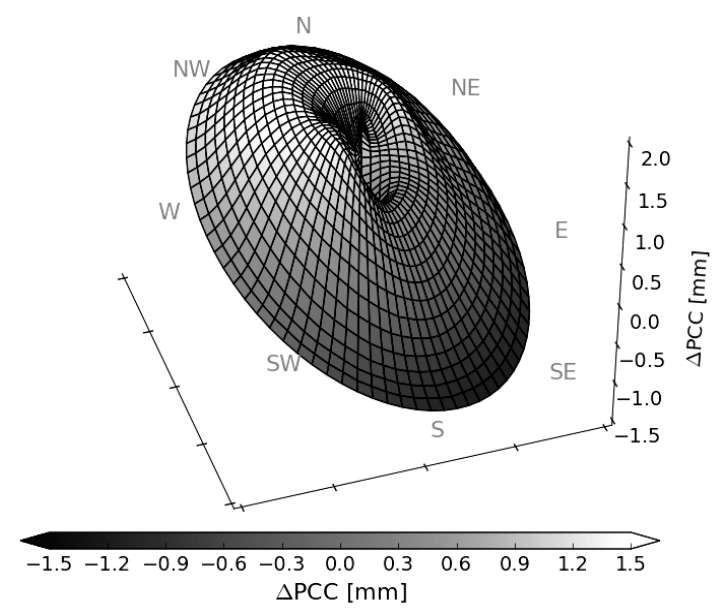

AV_RINGANT_G3T NONE316 (EPNC_14.ATX) - AV_RINGANTT_G3T NONE (igs08_1930.atx) frequency: G01

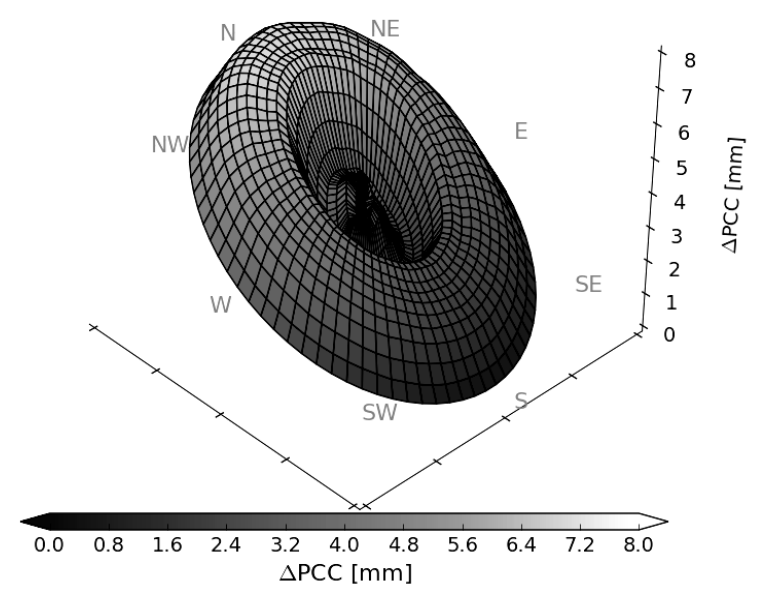

Fig. 5 Differences of Phase Center Corrections (PCC) for antennas mounted on POTS00DEU (left) and DARE00GBR (right) stations.

uniquely identifies two values: the vector from Antenna Reference Point (ARP) to the phase center point, which is called Phase Center Offset (PCO), and values depending on the satellite direction - Phase Center Variations (PCVs).

For the "ideal antenna", the phase center does not change its position (the antenna is identified with a point in the mathematical sense), but in practice the change of PCO or PCV can reach values from a few millimeters to even a few centimeters, which, if not modelled, is transferred directly to position error (or to baseline error, in case of differential positioning). This may change e.g. the scale, which occurred between the considered frames.

The antennas used at IGS and EPN reference stations usually have calibrations which are averaged from several absolute calibrations, so-called type mean calibrations. Alternatively, in the EPN network, some stations have calibrations which are performed individually for selected antennas using specialized robot or performed in chamber, so-called individual antenna calibrations. Compared to IGS08 standard, IGS14 includes calibrations for 17 additional antenna types, so that the absolute calibration rate has increased to over $90 \%$ (457 stations). Antennas without calibration remain only about $7 \%$ (36 stations). Moreover, in IGS14 the type mean calibrations of 19 antenna models were updated with respect to IGS08. This was possible thanks to the increasing availability of absolute calibrations for these antenna type-dome-combinations. For example, the calibration for LEIAR25 LEIT antenna model (as used in DARE00GBR) in IGS14 was derived from 92 antennas with absolute calibration whereas in IGS08 only 10 antennas were used. The Phase Center Correction (PCC, PCO + PCV) differences between
IGS14 and IGS08 for this model are in the range of -1.5 to $2 \mathrm{~mm}$ (Fig. 5, right). Moreover, in EPN processing some stations switched from type mean to individual calibration models, such as Potsdam (POTS00DEU), which is equipped with a JAV_RINGANT_G3T NONE antenna (Fig. 5, left). This station, since 2011 (based on EPN SINEX files), is equipped with the same antenna, but in EPN solutions the type mean calibration from IGS08 was used up to 1934 GPS week. After this epoch the calibration was switched to individual one. The differences between this individual calibration and IGS08 calibration varies from 0 to $7 \mathrm{~mm}$. The impact of GNSS ground antenna calibration updates on IGS stations can cause coordinate differences up to 6 and $19 \mathrm{~mm}$ in horizontal and vertical components respectively. Thus, such a big effect cannot be omitted. It is worth to notice that differences presented in Figure 5 do not state which calibration is better.

Despite of the slight difference in the scale between ITRF2008 and ITRF2014 (0.02 ppb), the radial elements of all GPS satellite antennas and GLONASS antenna phase offsets (z-PCOs) had to be changed in IGS14 due to a number of model changes (Earth Radiation pressure, antenna thrust). In IGS14 (w.r.t. IGS08) Z-PCO of satellite antennas changes average about $-6 \mathrm{~cm}$, resulting in a change of IGS frame scale of about $+0.5 \mathrm{ppb}(+3 \mathrm{~mm})$ (Rebischung and Schmid, 2016).

As already described in section 2, we investigated the impact of new and updated antenna calibrations on the implementation of the ITRF in Europe. For doing this, we mixed the calibration files used and the reference frames, see Table 2. On the basis of cumulative solutions we performed sevenparameter Helmert transformation between the 
solution for the period 1 and period 2. The determined transformation coefficients are shown in Table 3 (transformations 2A-1A, 2B-1B, 2D-1D, 2C-1C). It is worth to notice that, independently of the adopted frame (IGb08 or IGS14), for the same antenna calibration, we obtained a similar translation for all components of telocentric coordinates $(1.30,-0.42$, $0.37) \mathrm{mm}$, scale $(0.21 \mathrm{ppb})$ and frame orientation. Network orientation implemented by the rotation part is similar for all solutions regardless of the antenna calibrations used, which results in the lack of influence of antennas on the orientation of the network. On the other hand, the scale difference between transformation $2 \mathrm{~B}-1 \mathrm{~A}$ and the other transformations in Table 3, which is $0.74 \mathrm{ppb}$, is noticeable. This results in an increase in the length of the baselines in the network (from 1 to even $10 \mathrm{~mm}$ ) when different antenna calibrations were used (for baseline from a dozen to several hundred kilometers). Also, the components of translation vary considerably when we analyzed solutions with different antenna calibrations. They are visible for the TX, TY and TZ components, for which the differences are about 5.85, 6.58 and $8.48 \mathrm{~mm}$, respectively. Such high values are reflected in the values of station coordinates. As it is shown in Figure 5, coordinate differences for different antenna calibrations are up to few millimeters, for both horizontal and vertical components. Analyzing the changes of POTS00DEU (Potsdam, DE) and DARE00GBR (Daresbury, UK) station coordinates, the systematic bias due to differences in translation is evident. However, when the same antenna calibration is used, this effect can be reduced to less than $1 \mathrm{~mm}$, as it is shown in Figure 4. Differences in scale and translational component $\mathrm{TZ}$ can be observed if we compare the transformation parameters obtained for the same antenna calibrations with parameters of transformation between ITRF2014 and ITRF2008 reference frames. This is probably caused by two reasons. The first one comes from the satellite ephemeris used in the study, which for the time interval from GPS weeks 1928 to 1933 was estimated in IGb08 frame, and from GPS weeks 1934 to 1937 in IGS14 frame. Various types of satellite antennas were used in the ephemeris processing, and these affected the scale of the frame. The second reason is the difference between the position of the frame origin realized by the GNSS technique, and cumulative solution, which was used to define the ITRF. These effects can be partly eliminated if satellite ephemerides are transformed into the same reference frame, e.g. ITRF2014/IGS14. However, the complete elimination of influence of changes satellite antenna calibrations on ephemeris estimation can be achieved by their reprocessing in a uniform reference frame and with new antenna calibrations. At the end we would like to refer to the problem of choosing the set of fixed reference stations. In the first part of the paper, we presented that changing location and number of fixed reference stations certainly changes the realization of same and even of consecutive ITRF solutions.

To evaluate the size of this effect on Helmert transformation parameters, we processed two additional transformations. The first one is between modified solutions $2 \mathrm{~A}$ and $1 \mathrm{~A}$ (III*), the second one between modified solutions $2 \mathrm{~B}$ and $1 \mathrm{~B}$ (IV*).The network in these solutions was constrained with 28 stations in IGb08 (1A and $2 \mathrm{~B}$ ) solutions and to 30 stations in IGS14 solutions (2A and 2B) respectively. Similarly to the previous analysis, we determined the parameters of Helmert transformation between the cumulative solutions covering the period between GPS weeks 1928-1933 and 1934-1937. Table 3 shows the parameter (transformation 2A-1A III* - IGb08, 2B-1B IV* - IGS14). The translation parameter values increase proportionally to the number of used fixed reference station. The scale and orientation of the network remains consistent with the changing number of fixed reference stations. TZ changes translate into coordinates, but they are at the tenth of a millimeter. However, in case of analyzing the effects of systematic errors on the ITRF realization, they may lead to misinterpretations. Therefore, the selection of local GNSS fixed reference stations must be preceded by a careful analysis of the stability of coordinates changes and observation errors.

\section{SUMMARY}

In January 2017 a new realization of the ITRS, ITRF2014 had been introduced. It is characterized by high consistency with the previous ITRF2008 solution. Despite the great consistency of the ITRF solution itself, the introduction of the new satellite and ground antennas phase center calibrations in IGS14 leads to non-negligible differences in station positions. The updated antenna phase center models cause a change in the scale of the network, which we estimated at around $0.7 \mathrm{ppb}$. Also the translation parameters are changed, for all translation components, while the network orientation remains unchanged. Scale changes cause changes in baselines length between the stations (in most cases we obtained increased length). We also showed that the variable number of fixed reference stations in the GNSS local networks affects only the translation of the frame, and the change of its parameters is correlated with the number of fixed reference stations.

Presented results are obtained based on the Bernese double difference GNSS processing, which assumes the use of constrained stations and fixed satellite ephemeris to estimate positions in ITRFyy. This becomes a problem where studies about the impact of different phase center calibration on position are carried out. Therefore, further research on this issue should concern the elaboration of the investigated network using PPP method (if obtained accuracy will be on the level of differential method), in which the reference frame is transferred to the 
points using only satellite ephemeris. Only in this way the quantitative and qualitative influence of the new IGS14 antenna calibration on the implementation of ITRF2008 in Europe can be investigated. However, based on the analysis carried out in this paper, it is clear that the only method that will effectively remove the discontinuities caused by the new antenna calibrations is the third reprocessing of archived GNSS observations.

\section{ACKNOWLEDGMENT}

This research was financed by the Faculty of Civil and Environmental Engineering of Gdansk University of Technology statutory research funds. Calculations were carried out at the Academic Computer Centre in Gdansk.

\section{REFERENCES}

Altamimi, Z., Collilieux, X., Legrand, J., Garayt, B. and Boucher, C.: 2007, ITRF2005: A new release of the International Terrestrial Reference Frame based on time series of station positions and Earth Orientation Parameters. J. Geophys. Res., 112, B09401. DOI: 10.1029/2007JB004949

Altamimi, Z. and Collilieux, X.: 2009, IGS contribution to ITRF. J. Geod., 83, 3-4, 375-383. DOI: $10.1007 / \mathrm{s} 00190-008-0294-\mathrm{x}$

Altamimi, Z., Collilieux, X. and Métivier, L.: 2011, ITRF2008: an improved solution of the International Terrestrial Reference Frame. J. Geod., 85, 8, 457-473. DOI: $10.1007 / \mathrm{s} 00190-011-0444-4$

Altamimi, Z.: 2012, Role and importance of the International Terrestrial Reference Frame (ITRF) for sustainable development. ICG-7: Seventh Meeting of the International Committee on GNSS, Beijing, China.

Altamimi, Z., Rebischung, P., Métivier, L. and Collilieux, X.: 2016, ITRF2014: A new release of the International Terrestrial Reference Frame modeling nonlinear station motions. J. Geophys. Res., Solid Earth, 121, 6109-6131. DOI: 10.1002/2016JB013098

Drewes, H.: 2009, Reference systems, reference frames and geodetic datum - Basic considerations. IAG Symposia, 133, Sideris, M. G. (Ed.): Observing our Changing Earth, 3-9.
Dach, R., Lutz, S., Walser, P. and Fridez, P. (Eds): 2015, Bernese GNSS Software Version 5.2. User manual, Astronomical Institute, University of Bern.

EUREF: 2016, Guidelines for the EPN Analysis Centres, http://www.epncb.oma.be/_documentation/guidelines/

Hugentobler, U.: 2005, Models in GNSS data analysis, COMET - Advances in GPS Data Processing and Modeling for Geodynamics, University College London.

Kaminski, P., Figurski, M., Szafranek, K. and Kroszczyński, K.: 2010, Frequency and phase analysis of daily reprocessed solutions from selected EPN stations relating to geological phenomena. Acta Geodyn. Geomater., 7, 3 (159), 281-293.

Rebischung, P.: 2011, Upcoming switch to IGS08/igs08.atx, IGSMAIL No. 6354, 7th March.

Rebischung, P., Altamimi, Z., Ray, J. and Garayt, B.: 2016, The IGS contribution to ITRF2014. J. Geod., 90, 7, 611-630. DOI: 10.1007/s00190-016-0897-6

Rebischung, P. and Schmid, R.: 2016, IGS14/igs14.atx: a new framework for the IGS products, AGU Fall Meeting, San Francisco, CA.

Seidelmann, P.K.: 1982, 1980 IAU Nutation: The Final Report of the IAU Working Group on Nutation, Celestial Mechanics, 27, 1, 79-106.

Szafranek, K., Bogusz, J. and Figurski, M.: 2013, Configuration of the reference stations as the element of national reference frame reliability. Acta Geodyn. Geomater., 11, 1(173), 5-15. DOI: 10.13168/AGG.2013.0050

Wöppelmann, G., Bouin, M.-N. and Altamimi, Z.: 2008, Terrestrial Reference Frame implementation in global GPS analysis at TIGA ULR consortium. Phys. Chem. Earth, 33, 3-4, 217-224. DOI: 10.1016/j.pce.2006.11 